ITC 4/50

Information Technology and Control

Vol. 50 / No. 4 / 2021

pp. $752-768$

DOI 10.5755/j01.itc.50.4.28105
Wire Rope Weak Defect Signal Processing Methods Based on Improved SVD and Phase Space Reconstruction

Received 2020/12/02

Accepted after revision 2021/04/28

\title{
Wire Rope Weak Defect Signal Processing Methods Based on Improved SVD and Phase Space Reconstruction
}

\section{Muchao Chen}

School of Computer Science, Wuhan University, Wuhan, and Information Management Center, Guangdong Communication Polytechnic, Guangzhou, 510650, China; e-mail: chenmuchao@whu.edu.cn

\section{Yanxiang He}

School of Computer Science, Wuhan University, Wuhan, 430072, China; e-mail: yxhe@whu.edu.cn

Corresponding author: yxhe@whu.edu.cn

Due to the complexity of the interference operation environment of wire rope, the detection signals are usually weak and coupled in time-frequency domain, which makes the defect difficult to recognize, while the signal characterizations in phase space are also needed to be studied. Combining the nonlinear dynamic feature identification theories, phase space characteristics and chaotic features of wire rope defect detection signals are mainly investigated in this paper. First, principles of phase space reconstruction method for wire rope detection signals are presented by the chaotic dynamic indexes calculation of embedded dimension and delay time. Second, the change trends of the correlation dimension, approximate entropy and Lyapunov index of different phase space reconstructed wire rope defect detection signals are studied through the nonlinear simulation and analysis. Finally, a phase space reconstruction algorithm based on improved SVD is proposed, and the new algorithm is also compared with traditional signal processing methods. These results obtained by 6 groups of experiments were also evaluated and compared by the parameters of signal-to-noise ratio (SNR) and phase space trajectory chart, which manifests that the improved algorithm not only can increase the weak detection signal SNR to about 2.3dB of wire rope effectively, but also demonstrate the feasibility of the proposed methods in application.

KEYWORDS: Singular value decomposition (SVD), Phase space reconstruction, Weak signal, Denoising method. 


\section{Introduction}

As one of the most frequently applied ferromagnetic objects in practical engineering, steel wire rope plays an important role in loading in various fields such as, elevator, coal mine and ocean platform. However, the sophisticated working conditions and interferences make the defect detection signals very weak and mixed with strand and electromagnetic noises, which makes the wire rope defect, especially for the weak and inner damages such as the corrosion and wire break, difficult to inspect and distinguish. Besides, the common digital signal processing methods and weak defect recognition techniques, such as the lowpass filtering and single Hilbert transform, can hard to extract the weak defect features, and the chaotic characterization of wire rope defect detection signals also need to be investigated. As a consequence, the weak defect detection and signal processing are significant in safety guarantee and wire rope operation. Seeking for a valid and feasible weak signal processing method is also very important.

In the perspective of the dynamic characteristics research for wire rope detection system, researchers studied the nonlinear dynamics of the transverse, longitudinal and coupled vibration without external periodic driving forces for the wire rope in the friction hoist system by designing related experiments. They revealed the essence that the transverse vibration was a forced vibration while the longitudinal vibration was a complex random vibration state combining the time-frequency analysis for the vibration signals, and also pointed out that the vibration amplitude and strength deviation depended on the linear trend of the driving force frequency and characterized the nonlinear multi-order natural frequency features [8]. Italian scholars [1] studied the nonlinear dynamic models of the wire rope isolators on the basis of the Bouc-Wen model, and they revealed the frequency response rule of the wire rope under different vibrational excitation conditions through numerical simulations and experiments. Aiming at the problems of P-bifurcation in the noisy and bistable fractional-order system as well as the stochastic resonance, the case was mainly studied when the fractional order was within the interval of $(0,2]$, and the stochastic resonance characteristics within the range of upper and lower harmonic frequency were revealed by the nonlinear harmonic response theory [36]. Researchers [31] used the method of fractional order stochastic resonance and optimal fractional order calculations to achieve the effective identification and characterization for the original weak signals, and the validity of the fractional order method and its low-frequency weak signal detection characteristics were verified through the operation of numerical calculations and approximate analytical solution. In addition, based on the study of time-delay feedback monostatic and bistable stochastic resonance models, scholars [19] achieved the effective output of periodic low-noise and weak defect signals by selecting appropriate time-delay parameters, feedback strengths and computational steps, combining the band-pass filtering, improved power spectral kurtosis index and adaptive minimum-entropy based convolution model. Researchers [10] also proposed a multi-frequency and multi-scale based stochastic resonance detection model and method by applying the multi-stable stochastic resonance models of wavelet transform and parameter compensation, and when the amplitudes of the multi-scale and wavelet reconstructed signals were adjusted timely, the detectability of the weak low-frequency signals could be improved. Thus, the weak defect signals hid within the strong background noises could be extracted. Considering the non-stationary characteristics of the weak defect signals, when the time-frequency distribution under each decomposition scale was used as the modulation system and its transient energy distribution characteristics were studied, the effective information detection such as the amplitude and frequency of the multi-scale and frequency weak defect signals could be achieved eventually [11]. In the perspective of weak signal detection in steady-state system, Siliang Lu and Qingbo He [21] studied the underdamped, variable step and second-order stochastic resonance system deeply by selecting appropriate underdamped coefficients and the calculation steps, and achieved the optimal matched output for weak periodic signals under noisy environment conditions. Compared with traditional stochastic resonance system and methods, this proposed method has lower noise output after the second filtering, better bandpass filtering effect and anti-noise performance.

The emergence of the chaos theory has made the detection of weak signals under strong background noise conditions and the characterization of their 
dynamics a research hotspot. Since the duffing oscillators are mainly applied in the chaotic systems to detect weak signals [9, 13], researchers [30] investigated the bifurcation behavior of duffing oscillators and the feasibility of weak signal detection by taking advantage of the noise immunity of chaotic systems and the sensitivity of specific detection signals, and proposed a principle of periodic frequency-locked weak signal detection based on intermittent chaos. By using the chaotic systems evaluation indexes such as the Lyapunov index in the linear differential equation, the bifurcation threshold for weak signal chaos detection system can be determined. Moreover, according to Takens embedding theorem [34], the phase space reconstruction and modeling is the key of weak signal detection. Therefore, many researches in the aspect of embedded dimension calculation, model structure correction and parameter space optimization have been reported [25]. Based on the study of chaotic phenomenon, researchers [16] proposed a new trajectory decision function and discovered two phenomena of clockwise and counterclockwise stochastic resonance, where they achieved the detection of weak signals with unknown frequency components. Aiming at the defect detection of acoustic emission stress signals, researchers found that the defect gradually expanded, penetrated and fractured in the high stress stage, thus the acoustic emission was generated and higher stress energy was released, while the system output was presented as chaotic characteristic at the initial stage of stress loading, and it gradually became ordered as the loading process progresses and deepened $[20,17]$.

In the theoretical study of phase space reconstruction, researchers [27] proposed an improved defect growth and lifetime prediction model based on phase space distortions, combined with a multi-dimensional autoregressive model and a time-segmentation algorithm, the real-time residual lifetime prediction and defect diagnosis on multi-time scales were achieved. As for the nonlinear, acyclic and non-random vibration defect signals, the identification of defect states and key components of defect information were achieved by using pseudo-phase trajectories and correlation dimension analysis [37]. In the perspective of weak signal detection by duffing oscillator, researchers [22] carried out a comprehensive and systematic study on the noise effects in the behavior of periodic driving force dynamics and weak signal detection mechanism of duffing oscillators based on phase trajectory curves. From the perspective of the improvement and optimization of duffing oscillator model, researchers [38] proposed a van der Poel-duffing oscillator model to study the effects of noise intensity, detected signal frequency and phase shift on the output detected signal under strong background noise environment. Aiming at the limitation of the weak signal detection of the duffing system, the initial phase and periodic driving force influence laws are combined with Lyapunov index and other evaluation indexes to obtain the optimal threshold parameters for the dynamical state transition [35]. Besides, as the chaotic systems are deeply studied, scholars [33] proposed a Mathieu-Duffing oscillator detection model, which realizes weak signal detection under multiplication noisy conditions by solving the phase of Melnikov equation and detecting the transition amplitude between large-scale periodic motion and chaos weak signal detection. The feasibility of the duffing oscillator weak signal detection method based on cyclic boundary counting method and short-time Fourier transform state identification parameters was also investigated [12].

Due to the development of optimization theory and the deep study of nonlinear dynamics theory, researchers have successively proposed a weak signal detection method based on the sinusoidal chaotic attractor [24], which utilized Chua's circuit and chaotic synchronization characteristics, combining with adaptive and MUSIC algorithms to estimate the frequency and phase angle information of weak defect signals in strong noise environments, Finally, based on the phase difference between the reference signal and actual signal, effective detection and estimation of weak defect signal amplitude in secondary synchronous system was realized. In addition, scholars [26] proposed an unsaturated monostable stochastic resonance weak signal detection method based on the traditional model, and further improved the anti-noise performance of the model by the construction of the segmented monostable potential function model. As for the adjustment of stochastic resonance parameters, the adaptive parameter adjustment model and weak signal detection method [18] were investigated, and efficient estimation for the Kramers escape rate and other parameters are 
realized. Other optimization models and methods may include the strong coupled duffing-van der Poel oscillator, long and short term memory model for weak signal detection [32], the particle swarm optimization stochastic resonance model [14] and the approximate fractional integrator stochastic resonance model [15], which all further improved the accuracy and precision of weak signal detection in low SNR environments.

Besides, many optimization methods have also been proposed to reduce the computing resources and balance the exploration capability through the cooperative mechanism [28], differential evolution algorithms [5] and linear population size reduction strategy [29]. To overcome the slow convergence speed, poor global search ability, an improved quantum-inspired cooperative co-evolution algorithm based on combining the strategies of cooperative co-evolution and a new airport gate allocation optimization method were proposed in [2]. Besides, an improved differential evolution algorithm with neighborhood mutation operators and opposition-based learning developed in [3], as well as the differential evolution algorithm with wavelet basis function and optimal mutation strategy [5], the improved quantum evolutionary algorithms [6] and mixing multiple strategies [4], which all exhibit high accuracy and reliability as well as fast convergence speed when compared with other methods in parameter extracting and optimization, and showed great potential in solving complex airport gate assignment problems.

In order to solve the signal processing problems and challenges of weak defect detection in wire rope, the novelty and main contribution of this paper are as follows, first, the chaotic dynamic features of the original wire rope detection signals are analyzed from the perspective of phase space reconstruction, the calculation methods for embedded dimension and delay time are introduced. Afterwards, simulation analysis regarding to the reconstructed phase space and the evaluation indexes of wire rope weak signal such as the number of correlation dimension, approximate entropy and the maximum Lyapunov index are conducted, where the change rules of the chaotic dynamic characterizations from different wire rope testing signals are presented. Finally, a weak signal detection algorithm based on phase space reconstruction and improved SVD is proposed. The experimental veri- fication reveals the feasibility of the detection signal SNR and phase trajectory diagram in the weak defect signal characterizing and identifying under strong background noise conditions. Additionally, the limitations of the proposed method as well as the future work are discussed. The meaning of each variable appeared in the following sections are listed and explained in Table 1, such as,

Table 1

Meaning of each variable

\begin{tabular}{|c|c|c|c|}
\hline Variable & Meaning & Variable & Meaning \\
\hline $\mathrm{x}(\mathrm{i})$ & $\begin{array}{l}\text { Time series } \\
\text { signal }\end{array}$ & $\lambda$ & Threshold \\
\hline$y(i)$ & $\begin{array}{l}\text { Reconstructed } \\
\text { phase space }\end{array}$ & $\mathrm{H}$ & $\begin{array}{l}\text { Heaviside } \\
\text { function }\end{array}$ \\
\hline $\mathrm{y}^{\mathrm{N}}(\mathrm{i})$ & $\begin{array}{l}\text { Nearest neighbor } \\
\text { point of } y(i)\end{array}$ & $\mathrm{m}$ & $\begin{array}{l}\text { Dimension } \\
\text { number }\end{array}$ \\
\hline $\mathrm{R}_{\mathrm{m}}(\mathrm{i})$ & $\begin{array}{l}\text { Distance between } \\
\text { a point and its } \\
\text { neighbor }\end{array}$ & $\mathrm{n}$ & $\begin{array}{l}\text { Length of the } \\
\text { time series }\end{array}$ \\
\hline$\Delta \mathrm{R}$ & $\begin{array}{l}\text { Interval between } \\
\text { a point and the } \\
\text { next near neigh- } \\
\text { bor point }\end{array}$ & $\mathrm{N}$ & $\begin{array}{l}\text { Length of the } \\
\text { reconstructed } \\
\text { space }\end{array}$ \\
\hline $\mathrm{F}_{\mathrm{m}}(\mathrm{i})$ & $\begin{array}{l}\text { Percentage of the } \\
\text { near-neighbor } \\
\text { point }\end{array}$ & $\tau$ & Time delay \\
\hline $\mathrm{S}(\mathrm{m}, \mathrm{N}, \mathrm{r}, \mathrm{t})$ & $\begin{array}{l}\text { Autocorrelation } \\
\text { term of a time } \\
\text { series }\end{array}$ & Dim & $\begin{array}{l}\text { Correlation } \\
\text { dimension }\end{array}$ \\
\hline $\mathrm{C}(\mathrm{m}, \mathrm{N}, \mathrm{r}, \mathrm{t})$ & $\begin{array}{l}\text { Overall correla- } \\
\text { tion of a time } \\
\text { series }\end{array}$ & $\mathrm{S}(\mathrm{i})$ & $\begin{array}{l}\text { m-dimensional } \\
\text { vector }\end{array}$ \\
\hline $\mathrm{D}$ & $\begin{array}{l}\text { Embedded } \\
\text { dimension }\end{array}$ & $\mathrm{AE}(\mathrm{m}, \lambda)$ & $\begin{array}{l}\text { Approximate } \\
\text { entropy }\end{array}$ \\
\hline$\sigma$ & $\begin{array}{l}\text { Distance between } \\
\text { the initial } \\
\text { neighboring points }\end{array}$ & Ly & $\begin{array}{l}\text { Lyapunov } \\
\text { index }\end{array}$ \\
\hline $\mathrm{A}$ & $\begin{array}{l}\text { Decomposition } \\
\text { matrix }\end{array}$ & $\mathrm{U}$ & Left matrix \\
\hline S & $\begin{array}{l}\text { Singular value } \\
\text { matrix }\end{array}$ & V & Right matrix \\
\hline
\end{tabular}




\section{Principles and Methods}

According to the definition of embedded dimension introduced by Whitney et al., when there is a differentiable system of $\mathrm{S}$, there must also exists an embedded $\mathrm{S}$ that keeps its attractor the same topological structure as the dynamical system. Based on the above-mentioned inference, Takens et al. further expanded and proposed the idea of phase space reconstruction applying the time delay for univariate time series, which achieved the intuitive judgement for the system motion state by reconstructing the phase space trajectory. However, the parameter selection for time delay of $\tau$ and embedded dimension of $\mathrm{D}$ is the key to judge whether the system motion state is stable, periodic or chaotic from the phase space trajectory within the process of phase space reconstruction.

Likewise, when the phase space reconstructed dimension is smaller than the embedded dimension, the false near-neighbor point will be generated, which is caused by that the nonadjacent points in the attractors are overlapped in the process of dimension reduction mapping. Similarly, when the phase space reconstructed dimension number was bigger than the embedded dimension, the false projection or mapping would be produced on account of that the original adjacent points in the attractor start to keep away from each other in the process of dimension raising. Therefore, if the abrupt change just happens in the adjacent points of the attractor, the phase space dimension is equal to the embedded dimension. Specifically, assuming that the time series of $\mathrm{x}(\mathrm{i})$ could be reconstructed as a phase space,

$$
y(i)=[x(i), x(i+n), \ldots, x(i+(m-1) n)],
$$

where, $\mathrm{y}^{\mathrm{N}}(\mathrm{i})$ is the nearest neighbor point of the reconstructed phase space of $y(i)$,

$$
y^{N}(i)=\left[x^{N}(i), x^{N}(i+n), \ldots, x^{N}(i+(m-1) n)\right] .
$$

Then, the distance between a point and its neighbor in the reconstructed phase space is calculated as,

$$
R_{m}(i)=\left\|y(i)-y^{N}(i)\right\|=\sqrt{\sum_{j=1}^{m}\left[x(i+(j-1) n)-x^{N}(i+(j-1) n)\right]^{2}} .
$$

The distance between the next phase space reconstructed point and its neighbor is described as,

$$
\begin{aligned}
R_{m+1}(i) & =\sqrt{\sum_{j=1}^{m+1}\left[x(i+(j-1) n)-x^{N}(i+(j-1) n)\right]^{2}} \\
& =\sqrt{R_{m}(i)+\left[x(i+m n)-x^{N N}(i+m n)\right]^{2}}
\end{aligned}
$$

The interval between the phase space reconstructed point and the next near neighbor point is obtained as,

$$
\Delta R=\sqrt{R_{m+1}^{2}(i)-R_{m}^{2}(i)}=\left|x(i+m n)-x^{N N}(i+m n)\right| .
$$

To find the best embedded dimension, assuming that the critical points satisfy $R_{m+1}(i)=R_{m}(i)$, and the $R_{m}^{2}(i)$ is assumed to be the same as the mean diameter of the attractor, namely,

$$
\hat{R}_{m}{ }^{2}(i)=\frac{1}{N} \sum_{i=1}^{N}\left|x(i)-\frac{1}{N} \sum_{j=1}^{N} x(j)\right| .
$$

According to the definition of the false near neighbor point, the following condition should be satisfied when it occurred,

$$
F_{\mathrm{m}}(i)=\frac{\left|x(i+m n)-x^{N N}(i+m n)\right|}{\widehat{R}_{m}{ }^{2}(i)} \geq 10 \% .
$$

When $m$ is bigger than a certain of threshold and the percentage of the near-neighbor point of $F_{m}(i)$ is reduced to 0 and gradually remains stable, the threshold of $\mathrm{m}$ is the optimal embedded dimension (FNN method).

To eliminate the nonlinear dependence and correlation of the time delay calculation method to system variables, the C-C method can be considered. According to its principles, when the autocorrelation term of a time series $\mathrm{S}(\mathrm{m}, \mathrm{N}, \mathrm{r}, \mathrm{t})$ was eliminated, It could be expressed as,

$S(m, N, r, t)=\frac{1}{t} \sum_{i=1}^{t}\left(C_{i}(m, N / t, r, t)-C_{i}^{m}(1, N / t, r, t)\right)$,

where, the overall correlation $\mathrm{C}(\mathrm{m}, \mathrm{N}, \mathrm{r}, \mathrm{t})$ of the time series denotes the probability that the one-norm of the upper bound of a point in the time series of $\mathrm{s}_{\mathrm{i}}$ is not bigger than $r$. The specific description can be expressed as,

$$
C(m, N, r, t)=\frac{2}{M(M-1)} \sum_{1 \leq i<j \leq M} H\left(r-\left\|s_{i}-s_{j}\right\|\right),
$$

where, $\mathrm{M}=\mathrm{N}-(\mathrm{m}-1) \mathrm{t}$ denotes the number of points in the $\mathrm{m}$ dimensional embedded reconstructed phase 
space, $H$ represents the information entropy of the time series signal of $s(t)$, and when the time series length of $\mathrm{N}$ approaches to infinity, we can get,

$$
S(m, r, t)=\frac{1}{t} \sum_{i=2}^{t}\left(C_{i}(m, r, t)-C_{i}^{m}(1, r, t)\right) .
$$

If the time series signals are independent identically distributed and $\mathrm{r}_{\mathrm{i}}$ is selected appropriately,

$$
\Delta S(m, r, t)=\max \left[S\left(m, r_{i}, t\right)\right]-\min \left[S\left(m, r_{i}, t\right)\right] .
$$

The best delay time could be obtained when $\Delta \mathrm{S}$ reaches to a local minimum or crosses through the zero point for the first time, this method is particularly suitable for the calculation of delay time under a small amount of data condition in the wire rope defect detection.

Except for the reconstructed phase space dimension mentioned above, the correlation dimension is also an intuitive evaluation parameter that characterizes the attractor information of the inspected time series, and it is also an important index that reflects the freedom and complexity of a dynamic system. The earliest method of calculating the correlation dimension is the Gp method, which assumes that the correlation integral of a time series signal $\mathrm{S}_{\mathrm{i}}$ with a threshold of $\lambda$ is described as (which is similar to overall correlation mentioned above),

$$
C(\lambda)=\frac{2}{N(N-1)} \sum_{i=1}^{N} \sum_{\substack{j=1 \\ i \neq j}}^{N} H\left(\lambda-\left\|S_{i}-S_{j}\right\|\right)
$$

where, $\mathrm{H}$ denotes the Heaviside function, and $\left\|S_{i}-S_{j}\right\|$ is the Euclidean distance between the time series points of $S_{i}$ and $S_{j}$,

$$
H(s)=\left\{\begin{array}{ll}
0 & (s<0) \\
1 & (s>0)
\end{array} .\right.
$$

Therefore, when the threshold of $\lambda$ was chosen appropriately, the correlation dimension of Dim could be obtained.

$$
\operatorname{Dim}=\lim _{\lambda \rightarrow 0} \frac{\lg C(\lambda)}{\lg \lambda} .
$$

Actually, when the embedded dimension of $m$ was gradually increased and the slope in the linear section of the changing curves between $\mathrm{C}(\lambda)$ and $\lambda$ remained stable without any change, the embedded dimension is the correlation dimension of the reconstructed phase space.

Unlike the correlation dimension mentioned above, another important index in phase space reconstruction that measures the vector aggregation extent of high-dimensional space is the approximate entropy. Likewise, it can also evaluate the complexity of a time series system in a statistical mode. Assuming that the $\mathrm{m}$-dimensional vector composed of a time series signal x(i) was represented as S(i), namely,

$$
S(i)=[x(i), x(i+1), \ldots, x(i+m-1)] .
$$

The distance between two different vectors of $\mathrm{x}(\mathrm{i}-\mathrm{k})$ and $\mathrm{x}(\mathrm{j}-\mathrm{k})$ is,

$$
D[S(i), S(j)]=\max _{k=0,1, \ldots, m-1}|x(i+k)-x(j+k)| .
$$

Similarly, when the threshold parameter of $\lambda$ is appropriately set and the numbers of the distance less than $\lambda$ is counted up for a m-dimensional reconstructed phase space system, the reciprocal probability could be obtained and calculated as a ratio of,

$$
R_{i}^{m}(\lambda)=\frac{N o \cdot\{D(S(i), S(j))<\lambda\}}{N-m+1} .
$$

The logarithmic mean value is further calculated as,

$$
\Phi^{m}(\lambda)=\frac{\sum_{i=1}^{N-m+1} \ln R_{i}^{m}(\lambda)}{N-m+1}
$$

The final formula of the approximate entropy is obtained as,

$$
A E(m, \lambda)=\Phi^{m}(\lambda)-\Phi^{m+1}(\lambda)
$$

The Lyapunov index is an important evaluation parameter that measures the stability of a system state or orbital state in phase space reconstruction. Assuming that the mapping of a one-dimensional and discrete time series of $s_{0}$ is $F\left(s_{0}\right)$, after several iterations and mappings, two initially adjacent states are gradually separated in the chaotic motion, and their mapping space states as well as the iteration process can be represented as, 


$$
\begin{aligned}
s_{0} \stackrel{\sigma}{\leftrightarrow} s_{0}+\sigma & \Rightarrow F\left(s_{0}\right) \stackrel{\sigma e^{L y}}{\rightarrow} F\left(s_{0}+\sigma\right) \\
& \Rightarrow F^{2}\left(s_{0}\right) \stackrel{\sigma e^{2 L y}}{\rightarrow} F^{2}\left(s_{0}+\sigma\right) \\
& \ldots \\
& \Rightarrow F^{n}\left(s_{0}\right) \stackrel{\sigma e^{n L y}}{\rightarrow} F^{n}\left(s_{0}+\sigma\right)
\end{aligned}
$$

where, $\sigma$ is the distance between the initial neighboring points and Ly is the average separation index in every iteration. After $n$ times of iteration and mapping, the distance between two adjacent points becomes,

$$
\sigma e^{n L y\left(s_{0}\right)}=F^{n}\left(s_{0}+\sigma\right)-F^{n}\left(s_{0}\right) .
$$

Considering the limit situation, that is to say, $n \rightarrow \infty, \sigma \rightarrow 0$, then we can get,

$$
\begin{aligned}
\operatorname{Ly}\left(s_{0}\right) & =\lim _{n \rightarrow \infty} \lim _{\sigma \rightarrow 0} \frac{1}{n} \ln \left|\frac{F^{n}\left(s_{0}+\sigma\right)-F^{n}\left(s_{0}\right)}{\sigma}\right| \\
& =\lim _{n \rightarrow \infty} \frac{1}{n} \ln \left|\frac{d F^{n}(s)}{d s}\right|_{s=s_{0}}
\end{aligned} .
$$

Observed from Eq. (2.22), it could be deduced that in the limit case, $\operatorname{Ly}\left(\mathrm{s}_{0}\right)$ is independent of the initial value $\mathrm{s}_{0}$. Thus, Eq. (2.22) can be further simplified as,

$$
L y=\lim _{n \rightarrow \infty} \frac{1}{n} \sum_{i=0}^{n} \ln \left|\frac{d F}{d s}\right|_{s=s_{i}} .
$$

Thus, the Lyapunov index is obtained as Ly. On the other hand, when $n=1$, the distance between two adjacent points in the phase space satisfies,

$$
\sigma(t)=\sigma(0) e^{L y t} \text {. }
$$

Similarly, we can get,

$$
L y=\lim _{t \rightarrow \infty} \lim _{\sigma(0) \rightarrow 0} \frac{1}{t} \ln \left|\frac{\sigma(t)}{\sigma(0)}\right| .
$$

When the phase space is n-dimensional, the Lyapunov index can be expressed as,

$$
L y_{i}=\lim _{t \rightarrow \infty} \lim _{\sigma(0) \rightarrow 0} \frac{1}{t} \ln \left|\frac{\sigma_{i}(t)}{\sigma(0)}\right| .
$$

The maximum value of $\mathrm{Ly}_{\mathrm{i}}$ is usually taken as the maximum Lyapunov index. When the index is bigger than
0 , the distance between the adjacent points will gradually become larger, and the system will also gradually evolve into a chaotic state. When the index is less than 0 , the distance between the adjacent points will decrease and eventually evolve into a stable point, and the system can be judged as a stable periodic motion.

Hankel matrix transformation is the key of singular value decomposition (SVD) in various applications such as signal denoising, data compression. However, traditional single SVD can hardly satisfy the requirement of weak feature extraction and defect signal recognition under the background of multi-source interferences of wire rope detecting conditions. According to the above-mentioned calculation results of these evaluation indexes, it can be found that a single phase space reconstruction method is also difficult to achieve the dynamics features and weak defect signals recognition in wire rope defect detection. Therefore, an improved phase space reconstruction algorithm based on SVD denoising is considered to further investigate the dynamic system characterizations and defect recognition performance in the wire rope defect detection. Assuming that the Hankel matrix A is composed of the original wire rope defect detection signal of $\mathrm{s}(\mathrm{i})$, which can be represented as,

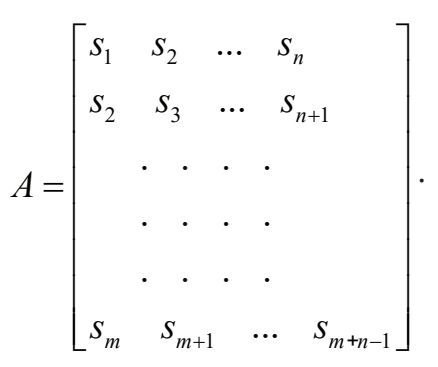

The further decomposition of matrix A $(m \times n)$ by SVD could be expressed as,

$$
A=U \cdot\left[\begin{array}{ll}
\Sigma & o \\
O & o
\end{array}\right] \cdot V^{T},
$$

where, $\mathrm{U}$ and $\mathrm{V}$ are orthogonal matrixes with the orders of $m$ and $n$, respectively, the singular value matrix decomposed from matrix $\mathrm{A}$ is a diagonal matrix with an order of $i$, where $i=\min (m, n)$ and o is a zero matrix. The diagonal matrix of $\sum$ composed of various eigenvalues can be expressed as, 
$\Sigma=\operatorname{diag}\left(\lambda_{1}, \lambda_{2}, \ldots, \lambda_{i}\right)$

The singular value decomposition for matrix A could be further simplified as,

$$
A=U * S * V^{T}=\sum_{k=1}^{i} A_{k}=\sum_{k=1}^{i} \lambda_{k} u_{k} v_{k}^{T}
$$

Where, $u$ and $v$ are the elements of the matrixes of $U$ and $V$, respectively, $\lambda$ is the singular value obtained from the SVD decomposition. Obviously, the decomposed singular value of $\lambda$ contains not only the information of the wire rope defect detection signal, but also the information of the interference noise. Generally, the original defect signal can be reconstructed by zero setting for the noise term in the singular value. However, the matrixes of $U$ and $V$ also contain part of the noise and useful defect signal information.

Therefore, an improved SVD denoising and phase space reconstruction combined wire rope defect detection and signal recognition method is proposed, the schematic diagram of the principles for the proposed algorithm and method is shown in Fig. 1.

Specific steps of the algorithm are as follows,

- First, the wire rope original signal of $S$ is reconstructed to form the multi-dimensional original detection data matrix of $\mathrm{A}$, then the reconstructed matrix of A is decomposed by SVD. Thus, the left decomposed orthogonal matrix of $U$, singular value matrix of S1 and the right orthogonal matrix of $\mathrm{V}$ are obtained, respectively.

- Second, in order to obtain more information of the defect detection signal and eliminate the noise interferences, the optimal left matrix of $\mathrm{U}$, singular value matrix of $\mathrm{S} 1$ and the right matrix of $\mathrm{V}$ can be obtained using the POD optimal algorithm, respectively. Then, the Hankel matrix of the original signal could be reconstructed and updated as A2, where the SNR of the new reconstructed signals are further compared. If it is less than a threshold of $\mathrm{k}$, the matrix reconstruction is continued again until that the new reconstructed signal SNR satisfies SNR $>\mathrm{k}$.

- Third, the C-C method and FNN method are applied to calculate the delay time and the embedded dimension of the reconstructed signals. Thereafter, the phase space of the SVD processed

\section{Figure 1}

Schematic diagram of an improved SVD-based phase space reconstruction algorithm

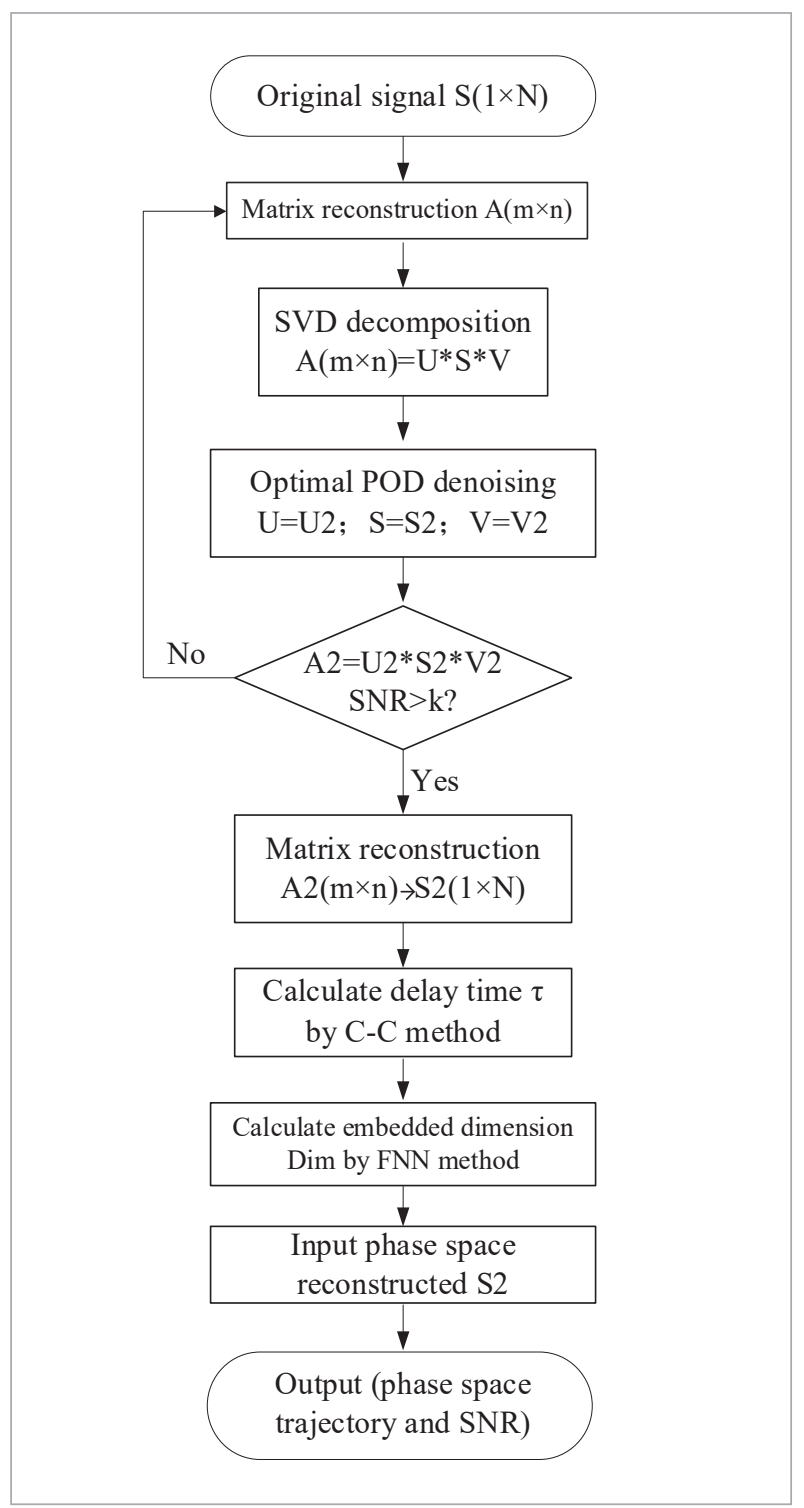

time series signal of $\mathrm{S} 2$ is reconstructed, and the trajectory diagram as well as the SNRs of the reconstructed phase space parameters are obtained. Finally, the wire rope defect detection signals and defect information could be identified according to these parameters and indexes.

As for the computation complexity, according to the schematic diagram of the proposed method shown in Fig. 1, it can be found that most of the computation 
is based on the calculation for 1D time series signal of $S(n)$ with a length of $n$, where the time complexity of the algorithm is $\mathrm{O}(\mathrm{n})$. However, when the time series signal were reconstructed into a $\mathrm{m} \times \mathrm{n}$ matrix, the time complexity is $\mathrm{O}(\mathrm{m} \times \mathrm{n})$. Then, further SVD transformation indicates that the matrix is decomposed into a multiplying form with the time complexity of $\mathrm{O}\left(\mathrm{m}^{2} \mathrm{n}\right)$, and the subsequent calculation steps in the proposed algorithms manifest that the reconstructed matrix of $\mathrm{S} 2(\mathrm{n})$ is mainly considered with a time complexity of $\mathrm{O}(\mathrm{n})$. Consequently, the overall computation complexity of the proposed method is the maximum value of $\mathrm{O}\left(\mathrm{m}^{2} \mathrm{n}\right)$.

\section{Simulations}

According to the wire rope detection signal characteristics, three different wire rope defect detection signals of $S_{1}, S_{2}$ and $S_{3}$ are mainly selected as the original data for phase space reconstruction, where each group of the defect data is composed of 10 small datasets (matrix data size $10 \times 1000$ ). Combining the principles of GP algorithm and the calculation results mentioned in Eq. (12) and Eq. (14), the relationship curves between the correlation integral and the near neighbor radius are obtained, as shown in Fig. 2(a). Thus, the correlation dimensions of different wire rope defect detection signals could be obtained by fitting the correlation integral curves and the original data. The calculation results of these correlation dimensions from three different wire rope detection signals and datasets are shown in Fig. 2(b).

It can be found that the correlation dimensions of three different wire rope defect detection signals and datasets are located between 2.2 and 3.8, and little fluctuations for the correlation dimensions from different datasets of signal 2 are observed, while lager fluctuations for the correlation dimensions of different detection datasets from signal 3 can be noticed, which indicates that the dynamic system of signal 3 has higher degrees of freedom and complexity.

Furthermore, according to the calculation principles of the approximate entropy and the above-mentioned results in Eq. (19), the approximate entropies for these three different wire rope detection signals $(3 \times 10$ groups of datasets) are obtained, as shown in Fig. 3. It can be concluded that these 10 datasets in

\section{Figure 2}

The calculation result for the correlation integral and correlation dimension. (a) A typical correlation integral curve for signal 1 and dataset 1 ; (b) Three different change rules of the correlation dimension for the original wire rope detection signals 1 to 3

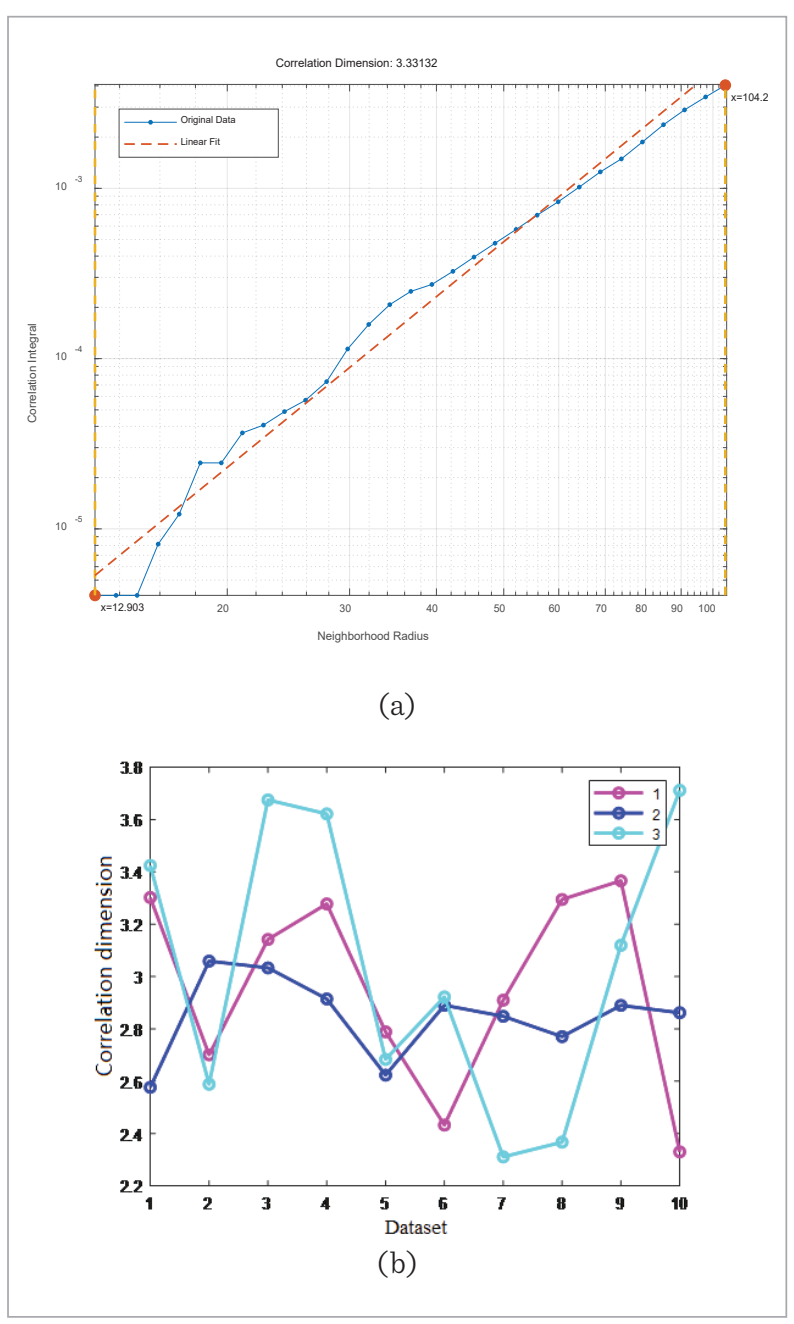

signal 3 all show relatively stable changing trends, while most of the approximate entropies calculated from the dataset in signal 1 are relatively larger. That is to say, the densities between different datasets are relatively higher and the time series is also more complex. However, only 5 tor7 approximate entropies obtained from the corresponding datasets in signal 2 are larger, which means that the probability of generating a new pattern in the dynamic motion is also higher.

Similarly, according to the principle of small data calculation aforementioned, the Maximal Lyapunov in- 


\section{Figure 3}

Approximate entropy calculation results for signals 1 to 3 with different datasets

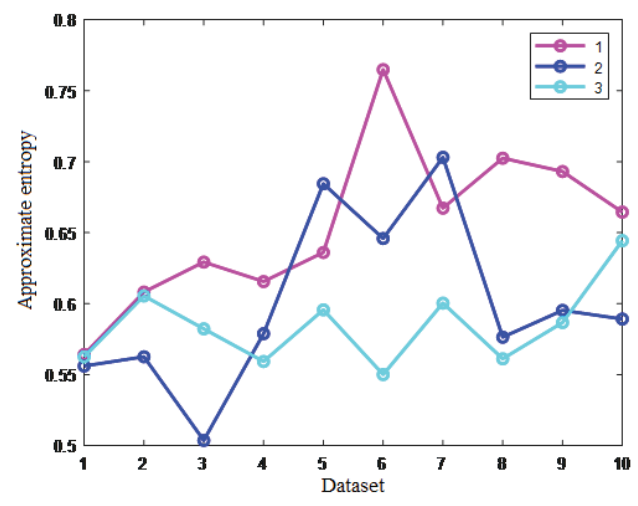

\section{Figure 4}

Results of the Lyapunov Index. (a) Log Lyapunov index curve; (b) Lyapunov index variation laws for three different detection original signals
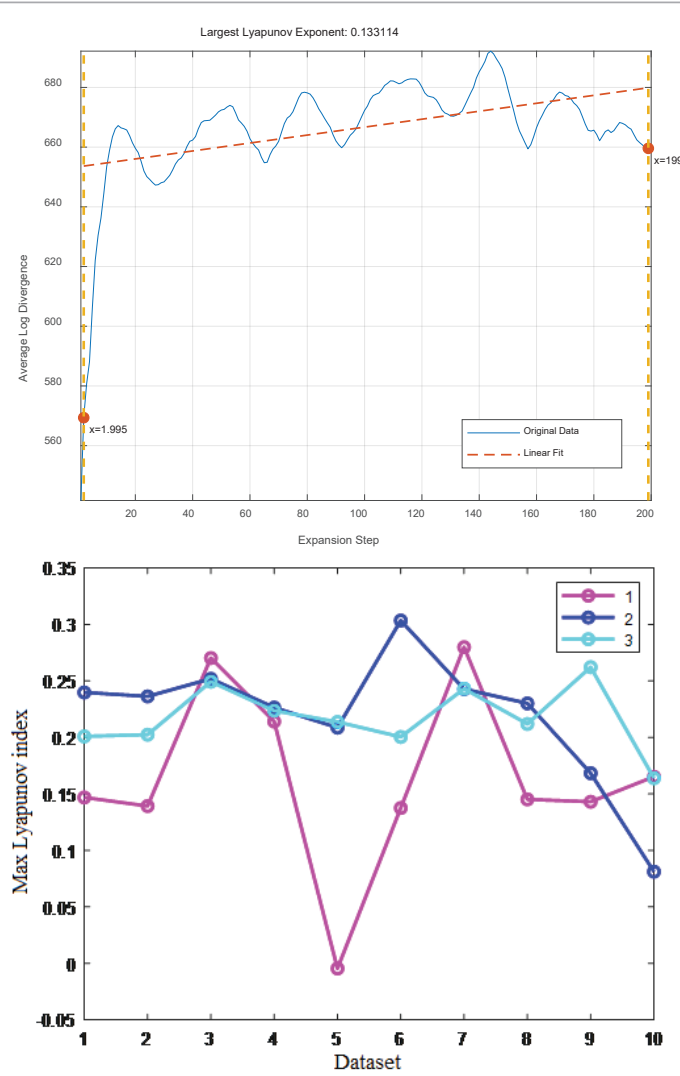

dex can be calculated from different datasets of three groups of wire rope defect detection signals, as shown in Fig. 4. Considering the Eq. (26), Fig. 4(a) shows the relationship curves between $\ln L_{j}(k)$ and $\mathrm{k} \Delta t$, and the maximum Lyapunov index can be obtained by the curve fitting method. It can also be observed that as the time interval or the step size increases, the logarithm of the Lyapunov index for signal 1 also increases in the initial stage and gradually fluctuates later. In addition, the further calculation results of the maximum Lyapunov index for three different wire rope detection signals and datasets are shown in Fig. 4(b). The results manifest that the maximum Lyapunov index fluctuates greatly among different datasets of signal 1, especially for dataset 5 , where the index is smaller and its dynamic state and chaotic orbit are more stable. While the other datasets are similar to that of the signal 2 and signal 3 datasets, and the Maximal Lyapunov index is in a small range of 0.1 to 0.35 , which also indicates that these datasets have better consistency.

\section{Experimental Results}

According to the principles of magnetic flux leakage (MFL) testing for wire rope defects, when a MFL testing device containing the magnetizing source of a pair of permanent magnets and the magnetic connecting bridge of a magnetic yoke composed of an armature is sanning along the axial direction of the specimen of wire rope with a diameter of $\Phi 28 \mathrm{~mm}$, and if any defect such as the wire broken, corrosion and abrasion is encountered, the magnetic flux within the body of the wire rope would be leaked due to the sudden change of the relative magnetic permeability in the position of the defect, as shown in Fig. 5. Consequently, the magnetic flux leakage can be captured through the mag-

Figure 5

Experimental apparatus and principles for signal acquisition and processing

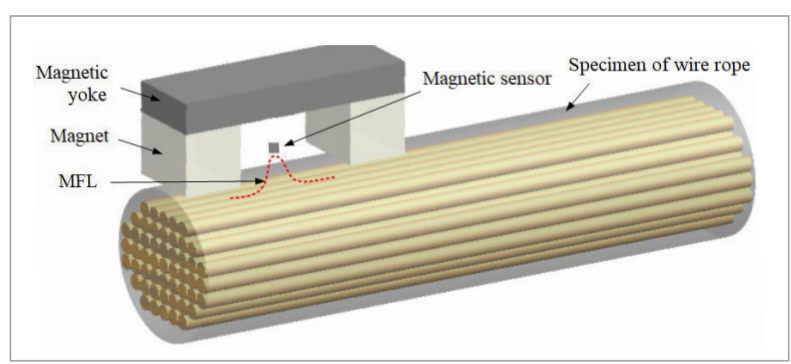


netic sensitive element of the sensors installed below the magnetic yoke with a lift-off distance of about 1-5 $\mathrm{mm}$. When the magnetic signals are transformed into voltage signals and transfered to the signal preprocessing circuit of lowpass filtering and the data acquisition (DAQ) modules, the original defect signals and information could be gotten. Actually, six groups of the original data for typical wire rope defect detection with different SNRs and defect information are obtained.

Furthermore, the comparison of the denoised signals calculated by the improved SVD based phase space reconstruction algorithm illustrated in Fig. 1 is shown in Fig. 6.

Explanatorily, the results shown in Fig. 6(a) to 6(f) are the comparison results of signal 1 to signal 6 of wire rope defect detection. The blue curve is the original wire rope defect detection signal obtained under different working conditions with various noises and interference of vibration, while the red curve is the corresponding wire rope defect detection signal processed by the improved algorithm. it can be observed that, the denoised signals processed by the improved SVD based algorithm features less baseline noise and interferences, which could be particularly found from the comparison results shown in Fig. 6(a), (b), (c) and (d). The further comparison results of the SNR, delay time and embedded dimension of the reconstructed phase space for wire rope defect detection signals before and after processed by the proposed algorithm are shown in Table 2.

\section{Table 2}

Comparison of the evaluation indexes before and after processed by the improved algorithm

\begin{tabular}{l|c|c|c|c|c|c}
\hline Detection signal & 1 & 2 & 3 & 4 & 5 & 6 \\
\hline SNR-before & -8.2 & -5.9 & -5.7 & -2.1 & 1.4 & -0.9 \\
\hline SNR-after & -6.7 & -5.9 & -3.9 & 1.4 & 3.7 & 1.4 \\
\hline Delay time-before & 5 & 5 & 4 & 3 & 5 & 7 \\
\hline Delay time-after & 5 & 10 & 5 & 4 & 5 & 4 \\
\hline $\begin{array}{l}\text { Embedded } \\
\text { dimension-before }\end{array}$ & 3 & 4 & 4 & 4 & 4 & 4 \\
\hline $\begin{array}{l}\text { Embedded } \\
\text { dimension-after }\end{array}$ & 4 & 3 & 4 & 4 & 4 & 4 \\
\hline
\end{tabular}

\section{Figure 6}

Comparison of signal processing results before and after the phase space reconstruction. (a) Detection signal 1;(b) Signal 2; (c) Signal 3; (d) Signal 4; (e) Signal 5; (f) Signal 6

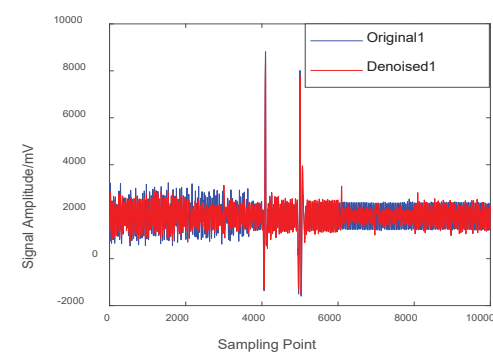

(a)

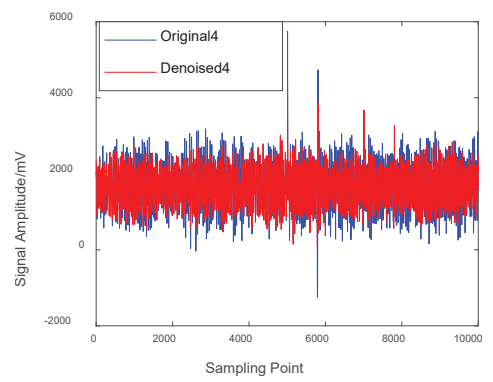

(d)

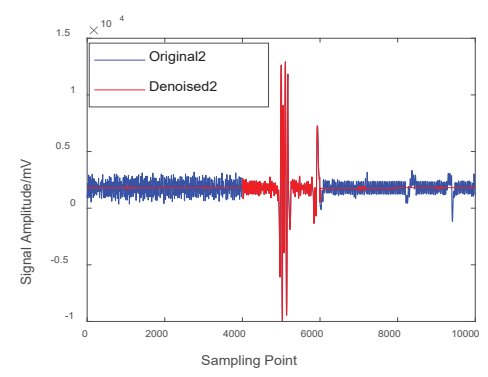

(b)

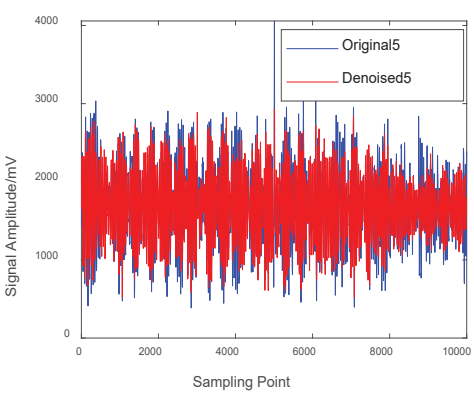

(e)

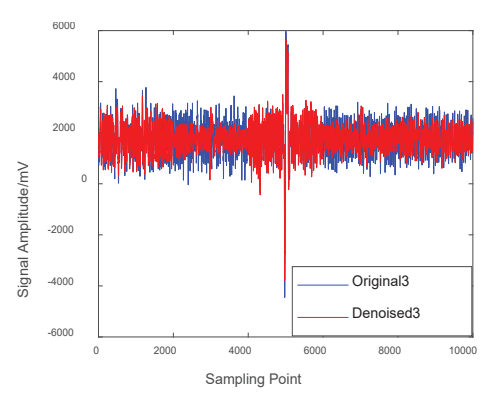

(c)

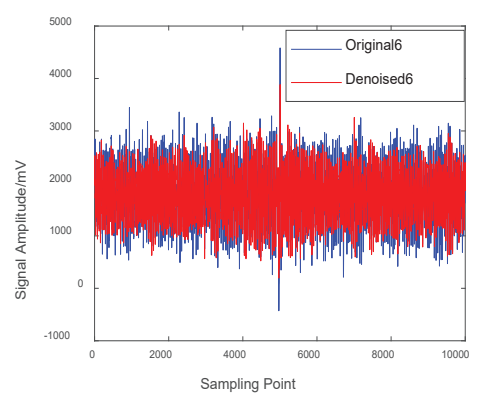

(f) 
It can be seen from Table 2 that the delay time of the reconstructed phase space processed by the improved algorithm has been increased from $5 \mathrm{~s}$ to $10 \mathrm{~s}$ for the detection signal 2 among the 6 groups of typical wire rope defect detection signals. While the embedded dimension of the phase space is reduced from 4 to 3 , and the SNR is increased from $-5.9782 \mathrm{~dB}$ to $-5.9741 \mathrm{~dB}$. As a comparison, the delay time of the reconstructed phase space for the detection signal 6 is shortened from $7 \mathrm{~s}$ to $4 \mathrm{~s}$, while the embedded dimension remains unchanged and the SNR of the detected signal processed by the improved algorithm is increased from $-0.9121 \mathrm{~dB}$ to $1.4757 \mathrm{~dB}$.

Besides, comparison results between different signal denoising methods such as the Empiric Mode Decomposition (EMD), lowpass (LP) and median filtering, Hilbert transform and wavelet denoising reported in related researhes were also compared, and the SNR calculation results were presented in Table 3.

It can be observed that, most of the decomposition components by EMD have lower SNRs compared with the original 6 groups of testing signals, except the component of Imf6. However, although Imf6 has bigger SNRs than than of the original testing signal, further signals of Imfs for these six groups of signals shown in Fig. 7 indicates that these Imf6 components can hardly be recognized in defect features.

\section{Table 3}

SNR comparison results by different signal denoising methods

\begin{tabular}{|c|c|c|c|c|c|c|c|}
\hline \multicolumn{2}{|c|}{$\begin{array}{l}\text { Detection } \\
\text { signal }\end{array}$} & 1 & 2 & 3 & 4 & 5 & 6 \\
\hline \multicolumn{2}{|c|}{ Original } & -8.2 & -5.9 & -5.7 & -2.1 & 1.4 & -0.9 \\
\hline \multirow{6}{*}{$\begin{array}{l}\text { EMD } \\
{[40]}\end{array}$} & Imf1 & -1.55 & -7.55 & -14.06 & -7.51 & -12.82 & -7.03 \\
\hline & $\operatorname{Imf2}$ & -7.44 & -3.94 & -6.38 & -5.99 & 0.16 & -4.23 \\
\hline & Imf3 & -4.36 & 8.26 & -8.33 & -3.03 & -10.05 & -1.23 \\
\hline & $\operatorname{Imf} 4$ & -1.94 & -1.57 & -3.46 & -8.07 & -6.83 & -6.81 \\
\hline & Imf5 & 2.21 & -1.68 & 0.99 & -0.85 & 1.79 & -4.99 \\
\hline & Imf6 & 21.88 & 0.18 & 7.88 & 7.11 & 20.32 & 2.69 \\
\hline \multicolumn{2}{|c|}{ LP [23] } & -8.25 & -5.97 & -5.60 & -1.27 & 1.56 & -0.32 \\
\hline \multicolumn{2}{|c|}{ Median [22] } & -10.82 & -6.53 & -9.64 & -0.25 & 0.69 & -4.05 \\
\hline \multicolumn{2}{|c|}{ Hilbert [39] } & -8.25 & -5.98 & -5.77 & -2.17 & 1.48 & -0.91 \\
\hline \multicolumn{2}{|c|}{ Wavelet [7] } & -10.37 & -3.05 & 0.67 & -11.35 & -15.02 & -1.11 \\
\hline
\end{tabular}

Figure 7

EMD components of Imf6 for six different wire rope testing signals

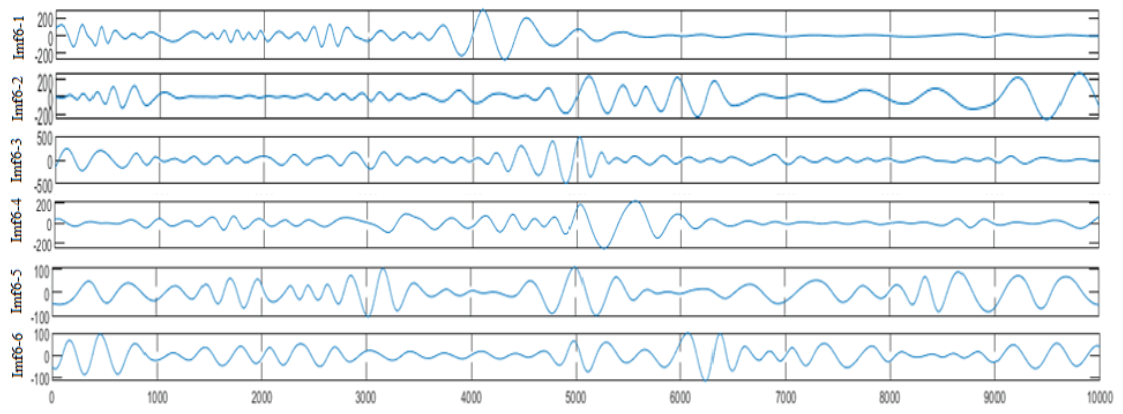

Similar results can also be found in the LP, median filtering signal, Hilbert transformed and wavelet denoisied signal SNR values. Namely, all of these signal processing methods are unavailable for weak wire rope signal detection and defect recognition.

On the other hand, the SNR comparison results ex- tracted from these 6 groups of typical wire rope detection signals before and after processed by the proposed algorithm are shown in Fig. 8.

Observed by the changing trend curves of SNRs shown in Fig. 8, it can be found that all the SNRs calculation results processed and denoised by the improved algo- 


\section{Figure 8}

SNR comparison results before and after processed by the improved algorithm

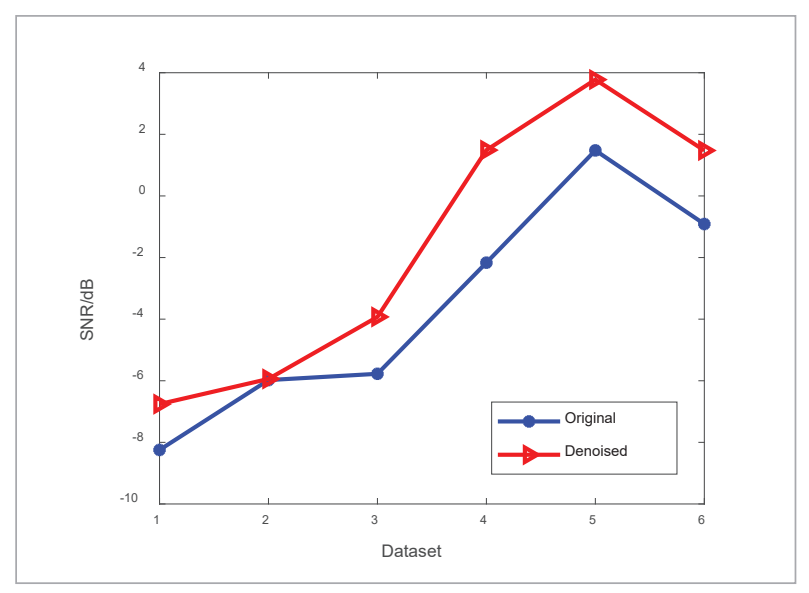

rithms are higher than that of these six groups of original signals for wire rope defect detection. Besides, the SNRs calculated from these six groups of signals processed by the improved algorithms all feature an increasing trend followed by a decrease gradient, as well as these original signals. In short, these SNRs calculation results demonstrated the effectiveness of the SVD based phase space reconstruction algorithm preliminarily, especially for its superiority in weak signal denoising.

On the other hand, in order to verify the phase space characteristics of the output detection signals, three groups of typical phase space trajectory curves of the wire rope detection signals before and after processed by the improved algorithm were calculated, respectively, as shown in Fig. 9. Specifically, Fig. 9(a), (c) and (e) are the phase space trajectory curves of the original wire rope detection signals, while Fig. 9(b), (d) and (f) are the calculation results processed by the improved algorithm. It can be found that the reconstructed phase space is symmetrically distributed, the diagonal part in the histogram is the phase space of the original signals, while the phase trajectories on these two sides are distributed with different delay times.

Comparing the results of the first group of wire rope detection signals shown in Fig. 9(a) and (b), it can be found that before the improved algorithm is applied, the embedded dimension of the original wire rope detection signal is 4 , the phase space trajectory is presented as a near-periodic state and mixed with many density points and interferences in the center location. After processed by the proposed improved algorithm, the embedded dimension of the phase space is 3, and the processed signals feature more prominent peaks. The defect information can be effectively and clearly identified, and the phase space trajectory curves are characterized with periodic states. Furthermore, less noise and chaos characteristics are mixed within the phase space trajectory, and a periodic attractor shape is exhibited. Similarly, although the other two groups of wire rope detection signals features stronger noise, and only one peak is shown in the original signal, the phase space trajectory is densely distributed, and the external phase trajectory curve is distributed discretely, as shown in Fig. 9(c) and (e). However, two peaks are expressed in the wire rope detection signals processed by the improved algorithm. Namely, two closely distributed defects can be effectively identified, and the external interference components in the phase space trajectories are also reduced, and the distribution pattern of the phase space trajectory center is more uniform, as shown in the Fig. 9(d) and (f). In conclusion, the phase space trajectory distribution results further demonstrated the effectiveness of the improved algorithm in wire rope defect detection signal processing and defect information recognition.

\section{Conclusion and Discussion}

Summarily, the concrete calculation methods for chaotic dynamics parameters of embedded dimension and delay time are introduced, and the chaotic dynamic characterizations of wire rope inspection signals are mainly analyzed and studied in this paper from the perspective of phase space reconstruction. Combining the calculation principles of different chaos evaluation parameters such as the correlation dimension, approximate entropy and the maximum Lyapunov index, the simulations are conducted regarding to three typical wire rope defect detection signals, where the characterizations of different wire rope inspection signals in the phase space were revealed. Naturally, an improved SVD and phase space reconstruction based wire rope weak signal detection and defect recognition method is proposed. Further- 


\section{Figure 9}

Phase space trajectory diagram before and after processed by the improved algorithm; (a) Results for original signal 1; (b) Results for processed signal 1; (c) Results for original signal 2; (d) results for processed signal 2; (e) Results for original signal 3; (f) Results for processed signal 3

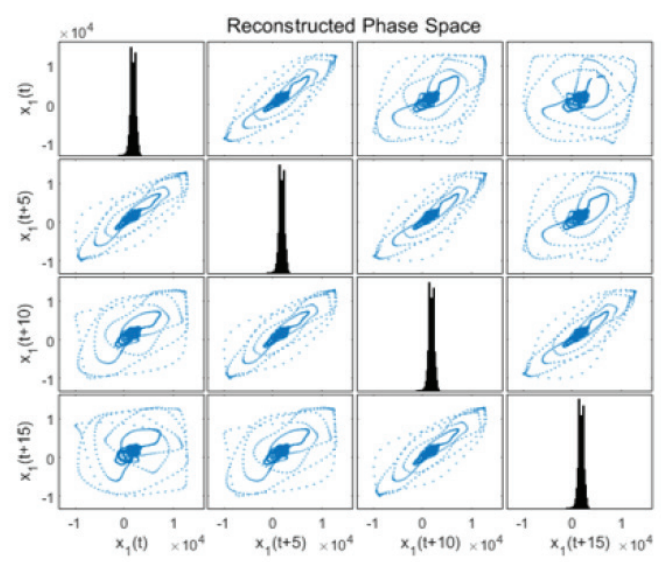

(a)

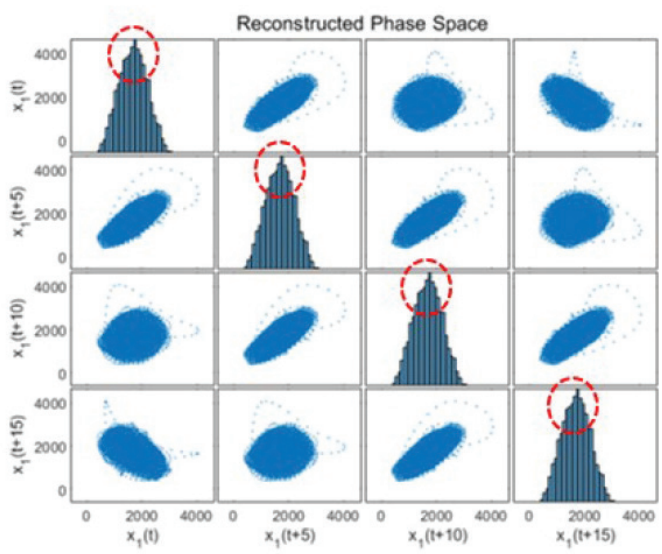

(c)

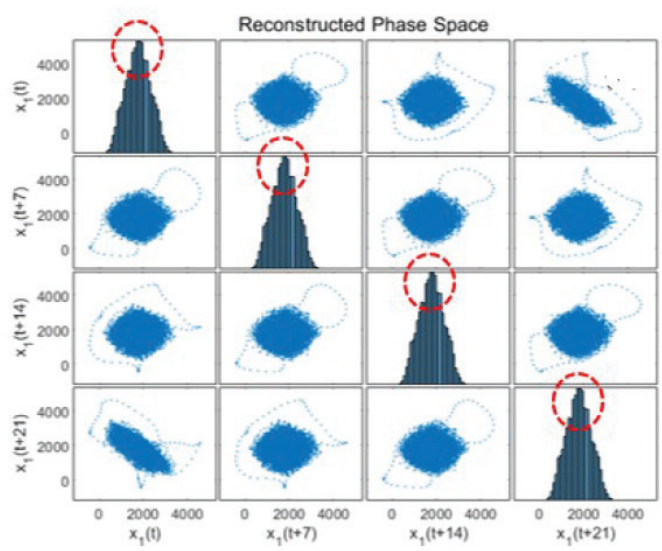

(e)

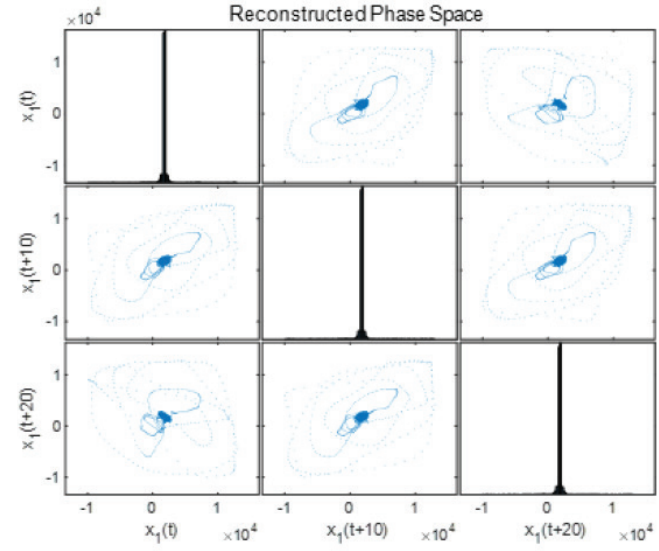

(b)

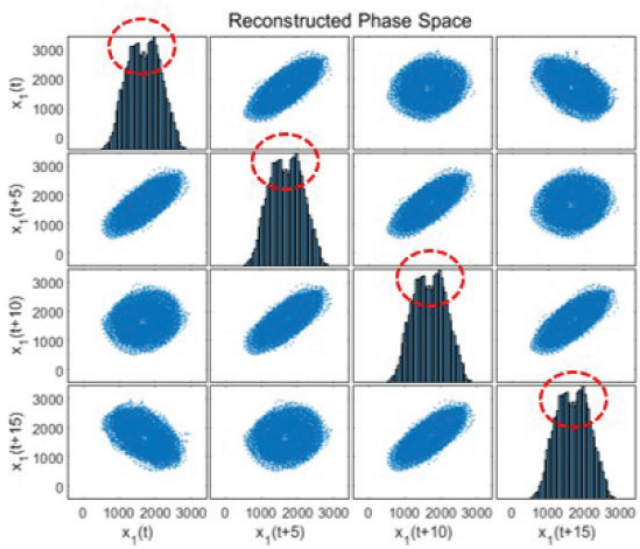

(d)

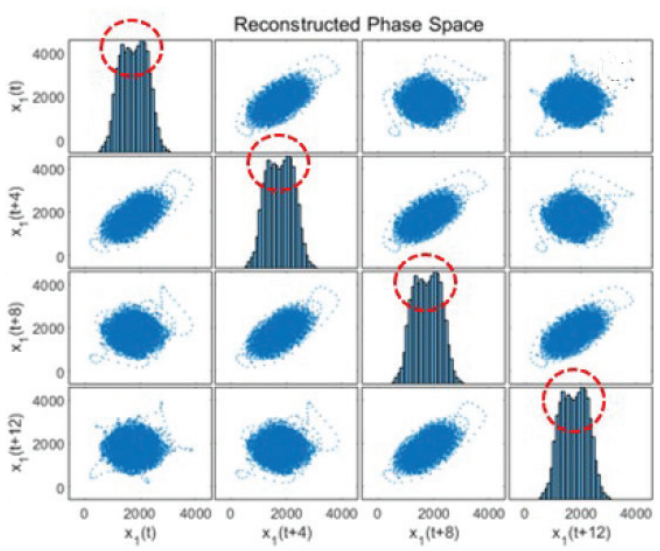

(f) 
more, the principle of the proposed algorithm and method are elaborated and explained by the comparison study. Moreover, the feasibility of the improved algorithm in the wire rope defect detection and weak signal recognition is also verified through the time and space domain, as well as the phase space trajectory analysis.

As for the limitations of the proposed method, it may lies in two aspects, one is that although the SNRs of the weak defect inspection signals are improved compared with the original signals, the interferences of

\section{References}

1. Barbieri, N., Barbieri, R., Silva, R., Mannala, M. J., de Sant'Anna Vitor Barbieri, L. Nonlinear Dynamic Analysis of Wire-rope Isolator and Stockbridge Damper. Nonlinear Dynamics, 2016, 86(1), 501-512.https://doi. org/10.1007/s11071-016-2903-1

2. Cai, X., Zhao, H., Shang, S., Zhou, Y., Deng, W., Chen, H., Deng, W. An Improved Quantum-inspired Cooperative Co-evolution Algorithm with Muli-strategy and Its Application. Expert Systems with Applications, 2021, 171, 114629. https://doi.org/10.1016/j.eswa.2021.114629

3. Deng, W., Shang, S., Cai, X., Zhao, H., Song, Y., Xu, J. An Improved Differential Evolution Algorithm and Its Application in Optimization Problem. Soft Computing, 2021, 1-22. https://doi.org/10.1007/s00500-02005527-x

4. Deng, W., Xu, J., Gao, X. Z., Zhao, H. An Enhanced MSIQDE Algorithm with Novel Multiple Strategies for Global Optimization Problems. IEEE Transactions on Systems, Man and Cybernetics: Systems, 2020. https:// doi.org/10.1109/TSMC.2020.3030792

5. Deng, W., Xu, J., Song, Y., Zhao, H. Differential Evolution Algorithm with Wavelet Basis Function and Optimal Mutation Strategy for Complex Optimization Problem. Applied Soft Computing, 2020, 106724. https://doi. org/10.1016/j.asoc.2020.106724

6. Deng, W., Xu, J., Zhao, H., Song, Y. A Novel Gate Resource Allocation Method Using Improved PSObased QEA. IEEE Transactions on Intelligent Transportation Systems, 2020. https://doi.org/10.1109/ TITS.2020.3025796

7. Gao, G., Qin, Y., Lian, M., Yue, L. Detecting Typical Defects in Wire Ropes Through Wavelet Analysis. Insight-Non-Destructive Testing and Condition Mon- strand and electromagnetic noises are still strong, which makes the weak defect hard to identify especially under extreme service conditions. Another shortcoming is that the shape and size of the reconstructed phase space may affect the validity and distinguishing results of the weak wire rope signal processing method when the chaotic dynamic indexes of delay time and embedded dimension are set unsuitably. Besides, how to further improve the real-time performance of the proposed method is also the future work.

itoring, 2015, 57(2), 98-105. https://doi.org/10.1784/ insi.2014.57.2.98

8. Guo, Y., Zhang, D., Zhang, X., Wang, S., Ma, W. Experimental Study on the Nonlinear Dynamic Characteristics of Wire Rope Under Periodic Excitation in A Friction Hoist. Shock and Vibration, 2020, 2020(2), 1-14. https://doi.org/10.1155/2020/8506016

9. Hénon, M. A Two-dimensional Mapping with a Strange Attractor. The Theory of Chaotic Attractors, Springer, New York, NY, 1976, 94-102. https://doi.org/10.1007/ BF01608556

10. Han, D., An, S., Shi, P. Multi-Frequency Weak Signal Detection Based on Wavelet Transform and Parameter Compensation Band-Pass Multi-Stable Stochastic Resonance. Mechanical Systems and Signal Processing, 2016, 70, 995-1010. https://doi.org/10.1016/j.ymssp.2015.09.003

11. He, Q., Wu, E., Pan, Y. Multi-Scale Stochastic Resonance Spectrogram for Fault Diagnosis of Rolling Element Bearings. Journal of Sound and Vibration, 2018, 420, 174-184. https://doi.org/10.1016/j.jsv.2018.01.001

12. Jia, R. L., Wang, K., Yan, X. P., Wang, K., Hou, J. Weak Signal Detection with Duffing Oscillator Based on Circular Boundary Counting Method and STFT State Identification Parameter. The 2nd International Conference on Electrical and Electronic Engineering, 2019. https://doi.org/10.2991/eee-19.2019.26

13. Kovacic, I., Brennan, M. J. The Duffing Equation: Nonlinear Oscillators and Their Behaviour. John Wiley \& Sons, 2011. https://doi.org/10.1002/9780470977859.ch6

14. Kumar, S., Jha, R. K. Weak Signal Detection from Noisy Signal Using Stochastic Resonance with Particle Swarm Optimization Technique. 2017 International 
Conference on Noise and Fluctuations, 2017. https:// doi.org/10.1109/ICNF.2017.7985952

15. Kumar, S., Jha, R. K. Weak Signal Detection Using Stochastic Resonance with Approximated Fractional Integrator. Circuits, Systems and Signal Processing, 2019, 38(3), 1157-1178. https://doi.org/10.1007/ s00034-018-0900-y

16. King, G. P., Stewart, I. Phase Space Reconstruction for Symmetric Dynamical Systems. Physica D: Nonlinear Phenomena, 1992, 58(1-4), 216-228. https://doi. org/10.1016/0167-2789(92)90110-9

17. Kong, X., Wang, E., Li, S., Lin, H., Xiao, P., Zhang, K. Fractals and Chaos Characteristics of Acoustic Emission Energy About Gas-bearing Coal During Loaded Failure. Fractals, 2019, 27(05), 1950072. https://doi. org/10.1142/S0218348X19500725

18. Lai, Z. H., Leng, Y. G. Generalized Parameter-Adjusted Stochastic Resonance of Duffing Oscillator and Its Application to Weak-signal Detection. Sensors, 2015, 15(9), 21327-21349. https://doi.org/10.3390/s150921327

19. Li, J, Li M, Zhang, J. Rolling Bearing Fault Diagnosis Based on Time-delayed Feedback Monostable Stochastic Resonance and Adaptive Minimum Entropy Deconvolution. Journal of Sound and Vibration, 2017, 401, 139-151. https://doi.org/10.1016/j.jsv.2017.04.036

20. Liu, J., Mao, J., Huang, B., Liu, P. Chaos and Reverse Transitions in Stochastic Resonance. Physics Letters A, 2018, 382(42-43), 3071-3078. https://doi.org/10.1016/j. physleta.2018.08.016

21. Lu, S., He, Q., Wang, J. A Review of Stochastic Resonance in Rotating Machine Fault Detection. Mechanical Systems and Signal Processing, 2019, 116, 230-260. https://doi.org/10.1016/j.ymssp.2018.06.032

22. Liu, S., Sun, Y., Jiang, X., Kang, Y. Comparison and Analysis of Multiple Signal Processing Methods in Steel Wire Rope Defect Detection by Hall Sensor. Measurement, 2021, 171, 108768. https://doi.org/10.1016/j.measurement.2020.108768

23. Liu, S., Sun, Y., Ma, W., Xie, F., Jiang, X., He, L., Kang, Y. A New Signal Processing Method Based on Notch Filtering and Wavelet Denoising in Wire Rope Inspection. Journal of Nondestructive Evaluation, 2019, 38(2), 1-14. https://doi.org/10.1007/s10921-019-0580-y

24. Li, G., Zhang, B. A Novel Weak Signal Detection Method via Chaotic Synchronization Using Chua's Circuit. IEEE Transactions on Industrial Electronics, 2016, 64(3), 2255-2265. https://doi.org/10.1109/ TIE.2016.2620103
25. Noakes, L. The Takens Embedding Theorem. International Journal of Bifurcation and Chaos, 1991, 1(04), 867-872. https://doi.org/10.1142/S0218127491000634

26. Qiao, Z., Lei, Y., Lin, J., Jia, F. An Adaptive Unsaturated Bistable Stochastic Resonance Method and Its Application in Mechanical Fault Diagnosis. Mechanical Systems and Signal Processing, 2017, 84, 731-746. https:// doi.org/10.1016/j.ymssp.2016.08.030

27. Qian, Y., Yan, R., Gao, R. X. A Multi-time Scale Approach to Remaining Useful Life Prediction in Rolling Bearing. Mechanical Systems and Signal Processing, 2017, 83, 549-567. https://doi.org/10.1016/j.ymssp.2016.06.031

28. Song, Y., Wu, D., Deng, W., Gao, X.-Z., Li, T., Zhang, B., Li, Y. MPPCEDE: Multi-population Parallel Co-evolutionary Differential Evolution for Parameter Optimization. Energy Conversion and Management, 2021, 228,113661. https://doi.org/10.1016/j.enconman.2020.113661

29. Song, Y., Wu, D., Wagdy Mohamed, A., Zhou, X., Zhang, B., Deng, W. Enhanced Success History Adaptive DE for Parameter Optimization of Photovoltaic Models. Complexity, 2021. https://doi.org/10.1155/2021/6660115

30. Wang, G., He, S. A Quantitative Study on Detection and Estimation of Weak Signals by Using Chaotic Duffing Oscillators. IEEE Transactions on Circuits and Systems Part IFundamental Theory and Applications, 2003, 50(7), 945-953. https://doi.org/10.1109/TCSI.2003.812606

31. Wu, C., Yang, J., Huang, D., Liu, H., Hu, E. Weak Signal Enhancement by Fractional-order System Resonance and Its Application in Bearing Fault Diagnosis. Measurement Science and Technology, 2019, 30(3), 035004. https://doi.org/10.1088/1361-6501/aaf904

32. Wang, K., Yan, X., Yang, Q., Hao, X., Wang, J. Weak Signal Detection Based on Strongly Coupled Duffing-Van der Pol Oscillator and Long Short-term Memory. Journal of the Physical Society of Japan, 2020, 89(1), 014003. https://doi.org/10.7566/JPSJ.89.014003

33. Wang, Q. B., Yang, Y. J., Zhang, X. Weak Signal Detection Based on Mathieu-Duffing Oscillator with Time-delay Feedback and Multiplicative Noise. Chaos, Solitons and Fractals, 2020, 137, 109832. https://doi.org/10.1016/j. chaos.2020.109832

34. Yue, L., Bao-Jun, Y., Li-Zhi, D., Ye, Y. The Bifurcation Threshold Value of the Chaos Detection System for a Weak Signal. Chinese Physics, 2003, 12(7), 714. https:// doi.org/10.1088/1009-1963/12/7/304

35. Yang, Y., Chen, J., Gao, Y., Fan, H., Yang, Y., Chen, J., Gao, Y., Fan, G. Detection of Weak Signal-to-Noise Ratio Signal While Drilling Based on Duffing Chaotic Oscil- 
lator. 2019 6th International Conference on Information Science and Control Engineering, 2019. https://doi. org/10.1109/ICISCE48695.2019.00207

36. Yang, J. H., Sanjuán, M. A. F., Liu, H. G., Litak, G., Li, X. Stochastic P-bifurcation and Stochastic Resonance in A Noisy Bistable Fractional-order System. Communications in Nonlinear Science and Numerical Simulation, 2016, 41(Dec.), 104-117. https://doi.org/10.1016/j. cnsns.2016.05.001

37. Zhu, Y.H., Li, C. W., Jin, W. D. Chaos Analysis of Fault Information of High-speed Train Based on Reconstructed Phase Space. 2016 35th Chinese Control Conference, 2016. https://doi.org/10.1109/ChiCC.2016.7554129
38. Zhihong, Z., Shaopu, Y. Application of Van Der Pol-Duffing Oscillator in Weak Signal Detection. Computers and Electrical Engineering, 2015, 41, 1-8. https://doi. org/10.1016/j.compeleceng.2014.11.007

39. Zhang, J., Tan, X., Zheng, P. Non-destructive Detection of Wire Rope Discontinuities From Residual Magnetic Field Images Using the Hilbert-Huang Transform and Compressed Sensing. Sensors, 2017, 17(3), 608. https:// doi.org/10.3390/s17030608

40. Zhang, J., Zheng, P., Tan, X. Recognition of Broken Wire Rope Based on Remanence Using EEMD and Wavelet Methods. Sensors, 2018, 18(4), 1110. https://doi. org/10.3390/s18041110 